\title{
UNIFIED WEAK-ELECTROMAGNETIC INTERACTIONS AND LEPTON-HADRON SYMMETRY - NINE LEPTONS AND NINE QUARKS ${ }^{\star}$
}

\author{
Yukio TOMOZAWA \\ Randall Laboratory of Physics, University of Michigan, Ann Arbor, MI 48109, USA
}

Received 1 June 1977

\begin{abstract}
A vector-like model with nine leptons and nine quarks is presented. Various consequences of the model are discussed.
\end{abstract}

Recent measurements of weak interaction phenomena indicate that a) there exists [1] a heavy lepton $\tau^{-}$ of mass $1.6-2 \mathrm{GeV}$ which decays into $\mu^{-}\left(\mathrm{e}^{-}\right)+$neutrals, b) the parity violation in heavy atoms [2] seems to be significantly smaller than the naive SalamWeinberg (S-W) model prediction [3, 4] while the neutrino reactions suggest the existence of parity violation in the hadronic dectors [5], and c) the "high-y anomaly" [6] in the $\bar{\nu}$ reaction and the ratio of the charged current cross section [7] by $\nu$ and $\bar{\nu}$ are indicative of the existence of righthanded heavy quarks in the charged current. Although none of these experimental evidences are conclusive at this time, the consideration of all these phenomena combined urges us to extend the original SW model [3].

In constructing a model, we adopt the following assumptions as guiding principles which have proved to be useful in past experience: i) universality or symmetry among leptons, such as the $\mu$-e universality and its generalization, and ii) symmetry between leptons and quarks. The latter symmetry, of course, can be true only modulo the color degree of freedom. Also it is assumed that iii) the departure from the SW model is minimal, by which we mean that the particles form doublets or singlets in the SU(2) $\times$ U(1) gauge group and the number of fields used is minimal.

Starting with lepton multiplets, conditions a) and i), along with the consideration of ordinary weak interaction phenomenology, dictate the existence of the left-handed doublets

$\left(\begin{array}{c}\nu_{\mathrm{e}} \\ \mathrm{e}^{-}\end{array}\right)_{\mathrm{L}},\left(\begin{array}{l}\nu_{\mu} \\ \mu^{-}\end{array}\right)_{\mathrm{L}},\left(\begin{array}{c}\nu_{\tau} \\ \tau^{--}\end{array}\right)_{\mathrm{L}}$.

\footnotetext{
* Work supported in part by the U.S. Energy Research and Development Administration.
}

The observation $b$ ) suggests that $\left(e^{-}\right)_{R}$ should be a member of a doublet. Introducing the heavy neutral leptons $E^{\circ}, M^{\circ}$, and $T^{\circ}$, we have right-handed doublets

$\left(\begin{array}{l}\mathrm{E}^{\circ} \\ \mathrm{e}^{-}\end{array}\right)_{\mathbf{R}},\left(\begin{array}{l}\mathrm{M}^{\circ} \\ \mu^{-}\end{array}\right)_{\mathbf{R}},\left(\begin{array}{l}\mathrm{T}^{\circ} \\ \tau^{-}\end{array}\right)_{\mathbf{R}}$.

These arrangements leave the left-out particles

$\left(\nu_{\mathrm{e}}\right)_{\mathrm{R}},\left(\nu_{\mu}\right)_{\mathrm{R}},\left(\nu_{\mathrm{e}}\right)_{\mathrm{R}} ;\left(\mathrm{E}^{\circ}\right)_{\mathrm{L}},\left(\mathrm{M}^{\circ}\right)_{\mathrm{L}},\left(\mathrm{T}^{\circ}\right)_{\mathrm{L}}$

to be assigned as singlets.

Now we come to the hadron sector. Since the leptons consists of 3 sets of triplet $\left(\mathrm{E}^{\circ}, \nu_{\mathrm{e}} \mathrm{e}^{-}\right)\left(\mathrm{M}^{\circ}, \nu_{\mu}, \mu^{-}\right)$ and $\left(\mathrm{T}^{\circ}, \nu, \tau^{-}\right)$, it is imperative to have 3 sets of quark triplet $(u, d, s),\left(u^{c}, d^{c}, s^{c}\right)$ and $\left(u^{t}, d^{t}, s^{t}\right)$ according to the symmetry requirement ii). We notice the difference in the charge structure however: the lepton triplets belong to the $\overline{3}$ representation and the quark triplets to the 3 representation of the SU(3) group. Analogous to the assignment for leptons, (I), (II) and (III) the quarks form doublets

$\left(\begin{array}{l}u \\ \tilde{d}\end{array}\right)_{L},\left(\begin{array}{l}u^{c} \\ \widetilde{s}\end{array}\right)_{L},\left(\begin{array}{l}u^{t} \\ d^{t}\end{array}\right)_{L} ;\left(\begin{array}{l}u \\ d^{c}\end{array}\right)_{R},\left(\begin{array}{l}u^{c} \\ s^{c}\end{array}\right)_{R},\left(\begin{array}{l}u^{t} \\ s^{t}\end{array}\right)_{R}$

and singlets

$d_{R}, s_{R}, d_{R}^{t} ; d_{L}^{c}, s_{L}^{c}$ and $s_{L}^{t}$.

In the above assignment, $\widetilde{d}$ and $\widetilde{s}$ are the Cabibbo rotated states of the $\mathrm{d}$ and $\mathrm{s}$ quarks, the more common nomenclature for heavy quarks is $u^{c} \equiv c, d^{c} \equiv b, u^{t}$ $\equiv \mathrm{t}$ etc.

The model to which we are led very naturally is an extension of the model considered by Fayet some years ago [8] and by Barnett [9] and Nieh [10] recent. ly. It is one of the vector-like models. The characteris- 
tics of the model are

(1) the neutral current of the charged leptons and the u-type quarks is pure vector, while the neutrinos (heavy neutral leptons) have a left (right) handed neutral current, and the negatively charged quarks have a mixed V, A, currents. More explicitly

$$
\begin{array}{r}
H_{\text {neutral }}=\mathrm{i} \frac{\mathrm{g}}{2} \frac{m_{z}}{m_{\mathrm{W}}} Z_{\mu}\left[-\overline{\mathrm{e}} \gamma_{\mu} \mathrm{e}\left(1-2 \sin ^{2} \theta_{\mathrm{w}}\right)\right. \\
+\frac{1}{2} \bar{\nu}_{\mathrm{e}} \gamma_{\mu}\left(1+\gamma_{5}\right) \nu_{\mathrm{e}}+\overline{\mathrm{u}} \gamma_{\mu} \mathrm{u}\left(1-\frac{4}{3} \sin ^{2} \theta_{\mathrm{W}}\right) \\
\left.-\frac{1}{2} \overline{\mathrm{d}}\left[\gamma_{\mu}\left(1-\frac{4}{3} \sin ^{2} \theta_{\mathrm{w}}\right)+\gamma_{\mu} \gamma_{5}\right] \mathrm{d}+\ldots\right]
\end{array}
$$

where $\theta_{\mathrm{W}}=\tan ^{-1} g^{\prime} / g$ is the Weinberge angle and

$$
\frac{g g^{\prime}}{\left(g^{2}+g^{\prime 2}\right)^{1 / 2}}=e, \frac{g^{2}}{8 m_{\mathrm{W}}^{2}}=\frac{G_{\mathrm{F}}}{\sqrt{2}}, \cos \theta_{\mathrm{W}}=\frac{m_{\mathrm{w}}}{m_{z^{\circ}}}
$$

as in the standard W-S model.

(2) The interaction hamiltonian (1) predicts $\sigma_{\nu \mu}^{\mathrm{e}}$ $=\sigma_{\bar{\nu}} \mathrm{e}$. At the present time, this is not incompatible with experiments [11], although the accuracy of the experiment is not good enough to support or exclude this kind of model.

(3) The existence of heavier quarks implies larger value for $R=\left(\mathrm{e}^{+} \mathrm{e}^{-} \rightarrow\right.$ hadrons $) /\left(\mathrm{e}^{+} \mathrm{e}^{-} \rightarrow \mu^{+} \mu^{-}\right)$than is measured currently. The asymptotic value of $R$ should be 7 .

(4) If there is no mixing between the heaviest quark triplet $\left(\mathrm{u}^{\mathrm{t}}, \mathrm{d}^{\mathrm{t}}, \mathrm{s}^{\mathrm{t}}\right)$ and the lower mass quarks $(\mathrm{u}, \mathrm{d}, \mathrm{s})$ and/or $\left(u^{c}, d^{c}, s^{c}\right)$, then the lowest mass state of hadrons which contain the t-type quarks becomes absolutely stable. Since this is an unlikely case in view of the past accumulated experiments (with cosmic rays and accelerators), it is more natural to expect some small amount of mixing, which allows t-quark states to decay. This may be considered as a generalization of the Cabibbo rotation between the $\mathrm{d}$ and $\mathrm{s}$ quarks. (The appearance of the $d$ and s quark fields as partners of $u$ and $u^{c}$ is a mystery. It would be more natural to identify $s \rightarrow d^{c}$ and $d^{c} \rightarrow s$ and to suppose that $d^{c}$ happens to be lighter than " $u$ " " and " $s$ " quarks.)

(5) If one finds more heavy leptons, one may want to add more quarks under the assumption of leptonhadron symmetry. These new quarks should be mixed with the lower quarks of the existence of an absolutely stable, massive hadron is to be avoided. This argument makes one feel that the addition of infinite series of lepton-hadron triplets is an unlikely possibility. Besides, the asymptotic free theory [12] admits no more than 5 (flavor) triplets of quarks.

(6) A recent measurement of trimuon production in the neutrino reaction [5] indicates that the three muons are produced in the lepton sector. This phenomenon is interpreted [13] as the production and cascade decays of a heavy charged lepton with mass $\sim 7 \mathrm{GeV}$. If that is the case, a fourth set of lepton and quark triplets would be required in our scheme. In order to avoid such an inflationary trend, the author proposes that the trimuon events involve the production of a charged Higgs boson [14] . Of course, that requires the existence of more than one Higgs scalar doublet. We notice that a model of the spontaneous $C P$ violation also requires Higgs scalar multiplets [15].

(7) The $\mu \rightarrow$ e $\gamma$ decay can occur via a mixing between $\mathrm{E}^{\circ}$ and $\mathrm{M}^{\circ}$, which looks analogous to a mixing of the $d$ and $s$ quarks, provided the masses of $E^{\circ}$ and $\mathrm{M}^{\circ}$ are different [16].

(8) The generation of the lepton and quark masses requires a detailed consideration of the Higgs coupling. For a single Higgs scalar doublet, the mixing for e.g. the $\left(\mathrm{M}^{\circ}, \nu_{\mu}, \mu^{-}\right)$triplet is expressed as

$\psi_{\mu}^{\mathrm{L}}=\left(\begin{array}{c}\nu_{\mu} \cos \varphi+\mathrm{M}_{0} \sin \varphi \\ \mu^{-}\end{array}\right)_{\mathrm{L}}$

$\psi_{\mu}^{\mathbf{R}}=\left(\begin{array}{c}-\nu_{\mu} \sin \xi+\mathrm{M}_{0} \cos \xi \\ \mu^{-}\end{array}\right)_{\mathrm{R}}$

for doublets and

$\chi_{\mu}^{\mathrm{R}}=\left(\nu_{\mu} \cos \xi+\mathrm{M}_{0} \sin \xi\right)_{\mathrm{R}} ;$

$\eta_{\mu}^{\mathrm{L}}=\left(-\nu_{\mu} \sin \varphi+\mathrm{M}_{0} \cos \varphi\right)_{\mathrm{L}}$

for singlets, where

$\sin \varphi \cos \xi=m_{\mu} / m_{0}$

$m_{0}$ being the mass of the heavy neutral lepton $\mathbf{M}_{0}$. The other triplets mix analogously. Since the absence of the right handed neutrino in the low energy weak interaction phenomena implies that $\xi \ll 1$, we obtain

$\sin \varphi=m_{\mu} / m_{0}$.

The coupling to the Higgs scalar is expressed as

$\lambda_{1}\left(\bar{\psi}_{\mu}^{\mathrm{L}} \varphi\right) \chi_{\mu}^{\mathrm{R}}+\lambda_{2}\left(\bar{\psi}_{\mu}^{\mathrm{R}} \varphi\right) \eta_{\mu}^{\mathrm{L}}+$ h.c.

where 
$\varphi=\left(\begin{array}{c}\varphi^{0} \\ -\varphi^{-}\end{array}\right)$

and the coupling constants of the Higgs scalar interactions are

$\lambda_{1}\left\langle\varphi^{\circ}\right\rangle=m_{0} \sin \varphi \sin \xi=m_{\mu} \tan \xi$

$\lambda_{2}\left\langle\varphi^{\circ}\right\rangle=m_{0} \cos \varphi \cos \xi \approx m_{0}$

i.e.

$\lambda_{1} / \lambda_{2}=m_{\mu} \tan \xi / m_{0} \ll m_{\mu} / m_{0}$

The eq. (9) implies that the Higgs coupling is dominated by the $\lambda_{2}\left(\overline{\mathrm{M}}_{0} \mathrm{M}_{0} \varphi^{\circ}\right) \approx\left(m_{0} /\left\langle\varphi^{\circ}\right\rangle\right) \overline{\mathrm{M}}_{0} \mathrm{M}_{0} \varphi^{\circ}$ term. This is a contrast to the standard W-S model where the Higgs coupling is $\left(m_{\mu} /\left\langle\varphi^{\circ}\right\rangle\right) \bar{\mu} \mu \varphi^{\circ}$. (There is no $\mu \mu \varphi^{\circ}$ coupling in a vector-like model.) The coupling strength of the decay $\varphi^{\circ} \rightarrow \mathrm{M}^{\circ} \overline{\mathrm{M}}^{\circ}$ is given by

$\frac{\lambda_{2}^{2}}{4 \pi}=\frac{1}{4 \pi} \frac{m_{0}^{2}}{\left\langle\varphi^{0}\right\rangle^{2}}=\frac{1}{4 \pi} \frac{1}{2} \frac{g^{2}}{m_{\mathrm{W}}^{2}} m_{0}^{2}=\frac{2 \sqrt{2}}{4 \pi} G_{\mathrm{F}} m_{0}^{2}$.

(9) Finally, we give a comment on the parity violation in atomic physics. By construction, the parity violation in a heavy atom should be at least a factor of $Z(\approx 80)$ smaller than the W-S prediction, since the electronic axial vector current is absent. From eq. (1), we obtain the parity violating interaction

$H_{\mathrm{pv}}=-\frac{G_{\mathrm{F}}}{\sqrt{2}} \overline{\mathrm{e}} \gamma_{\mu} \mathrm{e} \overline{\mathrm{d}} \gamma_{\mu} \gamma_{5} \mathrm{~d}\left(1-2 \sin ^{2} \theta_{\mathrm{w}}\right)$.

For hydrogen or deuterium atom [17] we have to compute the matrix elements $\left\langle\mathrm{p}\left|\overline{\mathrm{d}} \gamma_{\mu} \gamma_{5} \mathrm{~d}\right| \mathrm{p}\right\rangle$ and $\left\langle\mathrm{n}\left|\overline{\mathrm{d}} \gamma_{\mu} \gamma_{5} \mathrm{~d}\right| \mathrm{n}\right\rangle$. This can be done by using the SU(3) identity

$\overline{\mathrm{d} d}-\overline{\mathrm{s} s}=\overline{\mathrm{q}} \frac{1}{2}\left(-\lambda_{3}+\sqrt{3} \lambda_{8}\right) \mathrm{q}$

and the fact that sis does not contribute to the isovec. tor part $^{\ddagger}$;

$\left\langle\mathrm{p}\left|\overline{\mathrm{d}} \gamma_{\mu} \gamma_{5} \mathrm{~d}\right| \mathrm{p}\right\rangle-\left\langle\mathrm{n}\left|\overline{\mathrm{d}} \gamma_{\mu} \gamma_{5} \mathrm{~d}\right| \mathrm{n}\right\rangle=-(D+F) \overline{\mathrm{u}} \gamma_{\mu} \gamma_{5} \mathrm{u}$

where $D, A$ and $F$ are $\mathrm{d}$ and $\mathrm{f}$ couplings of the axial

\footnotetext{
* We use the formula for the SU(3) matrix calculation $\left\langle\mathrm{B}\left|\overline{\mathrm{q}} \lambda_{\mathrm{a}} \Gamma \mathrm{q}\right| \mathrm{B}\right)=\operatorname{tr}\left((D+F) \overline{\mathrm{B}} \lambda_{\mathrm{a}} \mathrm{B}+(D-F) \mathrm{B} \lambda_{\mathrm{a}} \overline{\mathrm{B}}\right) \mathrm{u}^{-} \Gamma \mathrm{u}$ where $B, \bar{B}$ are the standard $3 \times 3$ SU(3) matrix of the baryon octet.
}

vector current $(D=0.825$ and $F=0.425)$. If one assumes that $\left\langle\mathrm{p}\left|\overline{\mathrm{s}} \gamma_{\mu} \gamma_{5} \mathrm{~s}\right| \mathrm{p}\right\rangle=\left\langle\mathrm{n}\left|\overline{\mathrm{s}} \gamma_{\mu} \gamma_{5} \mathrm{~s}\right| \mathrm{n}\right\rangle \approx 0$, then one gets the matrix elements separately,

$$
\begin{aligned}
& \left\langle\mathrm{p}\left|\overline{\mathrm{d}} \gamma_{\mu} \gamma_{5} \mathrm{~d}\right| \mathrm{p}\right\rangle \approx\left\langle\mathrm{p}\left|\overline{\mathrm{q}} \frac{1}{2}\left(-\lambda_{3}+\sqrt{3} \lambda_{8}\right) \mathrm{q}\right| \mathrm{p}\right\rangle \\
& \quad=-(D-F) \overline{\mathrm{u}} \gamma_{\mu} \gamma_{5} \mathrm{u}
\end{aligned}
$$

and

$$
\begin{aligned}
& \left\langle\mathrm{n}\left|\overline{\mathrm{d}} \gamma_{\mu} \gamma_{5} \mathrm{~d}\right| \mathrm{n}\right\rangle \approx\left\langle\mathrm{n}\left|\overline{\mathrm{q}} \frac{1}{2}\left(-\lambda_{3}+\sqrt{ } 3 \lambda_{8}\right) \mathrm{q}\right| \mathrm{n}\right\rangle \\
& \quad=2 F \overline{\mathrm{u}} \gamma_{\mu} \gamma_{5} \mathrm{u} .
\end{aligned}
$$

The latter results are consistent with those given by Cahn and Kane [18].

It is a pleasure to thank $\mathrm{T}$. Witten for reading the manuscript.

\section{References}

[1] M.L. Pearl et al., Phys. Rev. Lett. 35 (1975) 1489; Phys. Lett. 63B (1976) 466; G.J. Feldman, Phys. Rev. Lett. 38 (1977) 117.

[2] P.E.G. Baird, Nature 264 (1976) 528; P.G.H. Sanders and E.N. Fortson, invited talks at the Washington meetings, 1977.

[3] S. Weinberg, Phys. Rev. Lett. 19 (1967) 1264; A. Salam, in: Elementary particle theory: relativistic groups and analyticity (Nobel Symposium No. 8), ed. N. Svartholm (Almquist and Wiksell, Stockholm (1968), p. 367.

[4] C. Bouchiat, invited talk to the topical conf. on Weak interactions (1976).

[5] B.C. Barish et al., Phys. Rev. Lett. 38 (1977) 577; A. Benvenuti et al., Phys. Rev. Lett. 38 (1977) 1110, 1183.

[6] A. Benvenuti et al., Phys. Rev. Lett. 36 (1976) 1478.

[7] A. Benvenuti et al., Phys. Rev. Lett. 37 (1976) 189.

[8] P. Fayet, Nucl. Phys. B78 (1974) 14.

[9] M. Barnett, Phys. Rev. D13 (1976) 671.

[10] H.T. Nieh, SUNY preprints (1977).

[11] H. Williams, invited talk at the particles and fields conference at BNL, 1976.

[12] D. Politzer, Phys. Rev. Lett. 26 (1973) 1346; Phys. Reports 14c (1974) 129; D. Gross and F. Wilczek, Phys. Rev. Lett. 26 (1973) 1343

[13] C.H. Albright et al., Phys. Rev. Lett. 38 (1977) 1187; V. Barger et al., Phys. Rev. Lett. 38 (1977) 1190; P. Langacker and G. Serge, Univ. of Penn. preprint UPR$0072 T$ (1977).

[14] Y. Tomozawa, UM HE 77-20 (1977). 
[15] T.D. Lee, Phys. Rev. D8 (1973) 1226; Phys. Reports 9c (1974) 143;

S. Weinberg, Phys. Rev. Lett. 37 (1976) 657.

[16] T.P. Cheng and Ling-Fong Li, Phys. Rev. Lett. 38 (1977) 381 .
[17] R.R. Lewis and W.L. Williams, Phys. Lett. 59B (1975) 70.

[18] R.N. Cahn and G.L. Kane, UM HE 77-1 (1977). 